\title{
Serum uric acid level is correlated with the clinical, pathological progression and prognosis of IgA nephropathy: an observational retrospective pilot-study
}

\author{
Pingfan Lu ${ }^{1}$, Xiaoqing Li ${ }^{1}$, Na Zhu ${ }^{1}$, Yuanjun Deng ${ }^{1}$, Yang Cai ${ }^{1}$, Tianjing Zhang ${ }^{1}$, Lele Liu ${ }^{1}$, Xueping Lin ${ }^{1}$, Yiyan \\ Guo ${ }^{1}$, Min Han ${ }^{\text {Corresp. } 1}$ \\ ${ }^{1}$ Division of Nephrology, Department of Internal Medicine, Tongji Hospital, Tongji Medical College, Huazhong University of Science and Technology, \\ Wuhan, China \\ Corresponding Author: Min Han \\ Email address: min_han@126.com
}

Objectives. This study was aimed to assess the relationship between serum uric acid (SUA) level and the clinical, pathological phenotype of IgA nephropathy (IgAN), and to determine the role of SUA level in the progression and prognosis of IgAN.

Methods. A total of 208 patients with IgAN were included in this study, which was classified into the normo-uricemia group and hyperuricemia group according to the SUA level. The clinical data at baseline, IgA Oxford classification scores (MEST-C scoring system), and other pathological features were collected and further analyzed. All patients were followed up and the prognosis was assessed using Kaplan-Meier survival curves. GraphPad Prism 7.0 and SPSS 23.0 were used for statistical analyses.

Results. In clinical indicators, patients with hyperuricemia had the significantly higher proportion of males to females, mean arterial pressure, the levels of total cholesterol, triglyceride, Scr, BUN, 24 hoururine protein, C3, and C4, the lower levels of high-density lipoprotein cholesterol and eGFR than those without $(p<0.05)$. In terms of pathological characteristics, the tubular atrophy/interstitial fibrosis scores, vascular injury scores, and glomerular sclerosis percentage were significantly higher in patients with hyperuricemia compared with those without $(p<0.01)$. There was no significant difference in the scores of mesangial hypercellularity, endocapillary hypercellularity, focal segmental glomerulosclerosis, as well as crescents between the two groups $(p>0.05)$. As for the depositions of immune complexes deposition in IgAN, the hyperuricemia group had less deposition of immunoglobulin $G$ and FRA than the normouricemia group $(p<0.05)$, while the deposition of immunoglobulin $A$, immunoglobulin $M$, and complement C3 in the two groups showed no statistical difference. The survival curve suggested that patients in the hyperuricemia group have significantly poorer renal outcome than those in the normo-uricemia group $(p=0.0147)$. Results also revealed that the SUA level is a valuable predictor of renal outcome in patients with IgAN. The optimal cutoff value was $361.1 \mu \mathrm{mol} / \mathrm{L}$ (AUC=0.76 \pm 0.08167 ) and $614 \mu \mathrm{mol} / \mathrm{L}$ (AUC $=0.5728 \pm 0.2029)$ for female and male, respectively.

Conclusions. The level of SUA is associated with renal function level and pathological severity of IgAN, and maybe a prognostic indicator of IgAN. 
1 Serum uric acid level is correlated with the clinical,

2 pathological progression and prognosis of $\lg A$

\section{3 nephropathy: an observational retrospective pilot-}

\section{4 study}

5 Pingfan $\mathrm{Lu}^{1}$, Xiaoqing $\mathrm{Li}^{1}$, Na Zhu ${ }^{1}$, Yuanjun Deng ${ }^{1}$, Yang Cai ${ }^{1}$, Tianjing Zhang, Lele Liu ${ }^{1}$,

6 Xueping Lin ${ }^{1}$, Yiyan Guo ${ }^{1}$, Min Han ${ }^{1}$

$8{ }^{1}$ Division of Nephrology, Department of Internal Medicine, Tongji Hospital, Tongji Medical

9 College, Huazhong University of Science and Technology, Wuhan, Hubei, China

Corresponding Author:

Min $\operatorname{Han}^{1}$

University of Science and Technology, 1095 Jiefang Avenue, Wuhan, Hubei, 430030, China

Email address: min_han@126.com

Objectives. This study was aimed to assess the relationship between serum uric acid (SUA) level

and the clinical, pathological phenotype of $\operatorname{IgA}$ nephropathy $(\operatorname{IgAN})$, and to determine the role of SUA level in the progression and prognosis of IgAN.

Methods. A total of 208 patients with IgAN were included in this study, which was classified into the normo-uricemia group and hyperuricemia group according to the SUA level. The clinical data at baseline, IgA Oxford classification scores (MEST-C scoring system), and other pathological features were collected and further analyzed. All patients were followed up and the prognosis was assessed using Kaplan-Meier survival curves. GraphPad Prism 7.0 and SPSS 23.0 were used for statistical analyses.

Results. In clinical indicators, patients with hyperuricemia had the significantly higher proportion of males to females, mean arterial pressure, the levels of total cholesterol, triglyceride, Scr, BUN, 24 hour-urine protein, C3, and C4, the lower levels of high-density lipoprotein cholesterol, and eGFR than those without $(\mathrm{p}<0.05)$. In terms of pathological characteristics, the 
tubular atrophy/interstitial fibrosis scores, vascular injury scores, and glomerular sclerosis percentage were significantly higher in patients with hyperuricemia compared with those without $(\mathrm{p}<0.01)$. There was no significant difference in the scores of mesangial hypercellularity, endocapillary hypercellularity, focal segmental glomerulosclerosis, as well as crescents between the two groups $(p>0.05)$. As for the depositions of immune complexes deposition in IgAN, the hyperuricemia group had less deposition of immunoglobulin G and FRA than the normouricemia group $(\mathrm{p}<0.05)$, while the deposition of immunoglobulin $\mathrm{A}$, immunoglobulin $\mathrm{M}$, and complement $\mathrm{C} 3$ in the two groups showed no statistical difference. The survival curve suggested that patients in the hyperuricemia group have significantly poorer renal outcome than those in the normo-uricemia group $(p=0.0147)$. Results also revealed that the SUA level is a valuable predictor of renal outcome in patients with IgAN. The optimal cutoff value was $361.1 \mu \mathrm{mol} / \mathrm{L}$ $(\mathrm{AUC}=0.76 \pm 0.08167)$ and $614 \mu \mathrm{mol} / \mathrm{L}(\mathrm{AUC}=0.5728 \pm 0.2029)$ for female and male, respectively.

Conclusions. The level of SUA is associated with renal function level and pathological severity of IgAN, and maybe a prognostic indicator of IgAN.

\section{Introduction}

IgA Nephropathy (IgAN), whose feature is the deposition of $\operatorname{IgA-dominant~in~the~mesangial~area,~}$ is the most prevalent primary glomerular disease in the world. Approximately $40 \%$ of patients almost completely lose their renal function within 30 years after diagnosis (Coppo \& D'Amico, 2005; D'Amico, 2004). Previous studies have demonstrated that clinical features, including severe proteinuria, poor renal function, and hypertension at the initial diagnosis are the predictors of poor outcomes in IgAN (Le et al., 2012; Barbour \& Reich, 2018; Mariani et al., 2018; Barbour $\&$ Reich, 2012). Histological features were also identified as crucial predictors of IgAN patients.

2 The Oxford classification of IgAN, also known as MEST score, including mesangial

3 hypercellularity (M), endocapillary hypercellularity (E), segmental glomerulosclerosis (S),

54 tubular atrophy/interstitial fibrosis $(\mathrm{T})$ has been shown to be of significant value in predicting the 
55

56

57

58

59

60

prognosis IgAN (Barbour et al., 2016; Maixnerova et al., 2016; Herzenberg et al., 2011a; Katafuchi et al., 2011; Coppo et al., 2014a; Lv et al., 2013). Recently, crescents (C) have been proposed to add to the Oxford classification of IgAN to form an updated MEST-C score system, which can provide a more comprehensive pathological prediction for the prognosis of IgAN (Trimarchi et al., 2017).

Numerous studies have shown that the level of uric acid can predict the incidence of atherosclerosis (Feig 2014; Gustafsson \& Unwin 2013; Li et al. 2014), hypertension (Wang et al. 2014), and coronary heart disease (Kim et al. 2010). Moreover, some studies have emphasized that high levels of serum uric acid (SUA) would form urates crystals that deposit in renal tubules and interstitial, leading to kidney fibrosis and failure (Su et al. 2020; Viggiano et al. 2018). Recently, some studies have found that there is a correlation between the SUA level and the progression of IgAN. A cohort study has shown that hyperuricemia is associated with the progression of IgAN, however, the effect of hyperuricemia on renal pathology was not evaluated (Bakan et al. 2015) in this study. The study conducted by Nagasawa et al. has shown that the SUA level is a predictor of IgAN in females but not in males (Nagasawa et al. 2016). Similarly, the evaluation of renal pathological changes was not included. Moriyama et al. have reported that hyperuricemia is a risk factor for the progression of IgAN with CKD stage G3a but not for stage G1, G2, or G3b-4. This study also has shown that, except for glomerulosclerosis percentage, there is no significant difference in the 2009 Oxford classification, crescentic percentage, and focal segmental sclerosis between the hyperuricemia group and the normo-uricemia group (Moriyama et al. 2015). Another study suggested that the relationship between hyperuricemia and IgAN progression was not very significant in patients with older age, lower eGFR, or interstitial lesion (Zhu et al. 2018). Similarly, this study did not use the updated Oxford classification of IgAN. These studies are partially inconsistent even contradictory and not comprehensive, cannot clarify the role of the SUA level in the progression and prognosis of IgAN. Therefore, it is imperative to conduct further research. 
82

83

84

In this retrospective study, we first used the updated Oxford classification criteria (MESTC), vascular injury, glomerulosclerosis, immunofluorescence score, clinical indicators, and prognosis analysis to comprehensively evaluate the relationship between the SUA level and the clinical, pathological characteristics of IgAN, to determine the impact of hyperuricemia on the development and prognosis of IgAN.

\section{Methods}

\section{Patients screening and Clinical Data Collection}

From January 2015 to May 2016, a total of 240 patients diagnosed with IgAN according to diagnostic criteria at Tongji Hospital were included in the study. The exclusion criteria of this study were listed as below: (1) the secondary IgAN caused by systemic diseases such as autoimmune disorders, chronic hepatitis, tumor, (2) incomplete clinical and pathologic data, or (3) the number of glomeruli in renal biopsy specimen less than eight. According to the above criteria, 32 patients were excluded and 208 patients were eventually involved in our research. The clinical data were collected at IgAN diagnosis. Blood samples were collected in the morning from fasting participants who had been directed to avoid eating any food that might affect the test result. The SUA levels $>420 \mu \mathrm{mol} / \mathrm{L}$ in men and $>360 \mu \mathrm{mol} / \mathrm{L}$ in women was defined as hyperuricemia. Based on the levels of SUA, there were 138 cases with hyperuricemia and 70 cases with normo-uricemia.

This study was approved by the Ethical Committee of Tongji Hospital (No.TJ-IRB20180608), and the Ethics Committee waived the need for informed consent from participants of this study.

\section{Histologic Evaluation}

Every renal biopsy specimen was scored by two pathologists who did not know the patient's clinical data. All of the renal specimens were classified and graded by five key pathological features: M, E, S, T, and C according to the MEST-C score of Oxford Classification(Trimarchi et 
106

107

108

109

110

111

112

113

114

115

116

117

118

119

120

121

122

123

124

125

126

127

128

129

al., 2017). Other pathological features, including glomerular global/ ischemic sclerosis, arteriosclerosis, arteriolar hyalinosis, and immune complex deposition were also described in all specimens. The histopathological grading schema we used was presented in Table 1 . When the two pathologists differed in their assessments, the biopsy specimen must be reviewed again until an agreement was reached.

\section{Statistical Analyses}

GraphPad Prism 7.0 and SPSS 23.0 were used for statistical analyses. Continuous and categorical data were presented as mean $\pm \mathrm{SD}$ and number $(\%)$, respectively. The comparison between the normo-uricemia group and the hyperuricemia group using the parametric t-test or Mann-Whitney. The overall renal survival rate of IgAN was presented using the Kaplan-Meier curve and the difference between the two groups was compared using the log-rank test. The $\mathrm{p}<$ 0.05 was regarded as statistical significance in all tests.

\section{Results}

\section{Demographic and clinical data}

The demographic and clinical data of 208 participants in this study were shown in Table 2 . The ages of all patients ranged from 15 to 63 years old, with an average of $33.8 \pm 10.7$ years old. Among them, $82(39.42 \%)$ were males, and 126 (68.68\%) were females. The mean arterial pressure of patients was $94.0 \pm 11.7 \mathrm{~mm} \mathrm{Hg}$, among which 23 patients had hypertension previously, $16(7.69 \%)$ had poor blood pressure control, and $22(10.58 \%)$ were taking antihypertensive medication. In this study, $14.42 \%$ of the patients had previously received ACEI and/or ARB treatment, and 4.81\% underwent tonsillectomy previously. For all patients, the mean levels of serum albumin, 24-h urine protein, SUA, serum creatinine (Scr), and eGFR were 39.48 $\pm 5.77 \mathrm{~g} / 1,1.35 \pm 1.58 \mathrm{~g} / \mathrm{d}, 348.00 \pm 109.10 \mu \mathrm{mol} / 1,89.89 \pm 41.49 \mu \mathrm{mol} / 1$ and $82.23 \pm 30.22$ $\mathrm{ml} / \mathrm{min} / 1.73 \mathrm{~m}^{2}$, respectively. 
130

131

132

133

134

135

136

137

138

139

140

141

142

143

144

145

146

147

148

149

150

151

152

153

154

155

156

\section{The comparison of clinical characteristics between the normo-uricemia group and the}

\section{hyperuricemia group}

The comparison of baseline characteristics between the normo-uricemia group and the hyperuricemia group was presented in Table 3. There were 70 patients $(33.65 \%)$ with hyperuricemia, including 36 males (51.43\%). These results were similar to the previous study conducted by Shi-Y (Shi et al., 2012). Results showed that the mean arterial pressure of patients with hyperuricemia was significantly higher than those without $(91.8 \pm 10.7 \mathrm{~mm} \mathrm{Hg}$ vs $98.2 \pm$ $12.5 \mathrm{~mm} \mathrm{Hg}, \mathrm{p}=0.0004)$. In terms of coagulation indicators, although the FDP level was higher in the hyperuricemia group $(3.23 \pm 0.74 \mathrm{mg} / \mathrm{lvs} 3.86 \pm 1.38 \mathrm{mg} / \mathrm{l}, \mathrm{p}=0.0006)$, the APTT level was similar in both groups $(\mathrm{p}=0.7833)$. As for blood lipids, hyperuricemia patients had an elevated level of total cholesterol, triglyceride, and decreased level of HDL-C $(\mathrm{p}=0.026, \mathrm{p}=$ $0.0005, \mathrm{p}=0.0056$, respectively). However, the LDL-C levels of the two groups were similar ( $\mathrm{p}>$ 0.05). This study also revealed the blood urea nitrogen $(5.12 \pm 1.52 \mathrm{mmol} / \mathrm{l}$ vs $6.49 \pm 1.95$ $\mathrm{mmol} / \mathrm{l})$, Scr $(80.40 \pm 40.58 \mu \mathrm{mol} / \mathrm{l}$ vs $109.00 \pm 36.54 \mu \mathrm{mol} / \mathrm{l})$ and $24-\mathrm{h}$ urine protein $(1.05 \pm$ $1.27 \mathrm{~g} / \mathrm{d}$ vs $1.81 \pm 1.67 \mathrm{~g} / \mathrm{d})$ in hyperuricemia patients were higher, while the eGFR (98.98 \pm $25.12 \mathrm{ml} / \mathrm{min} / 1.73 \mathrm{~m}^{2} \mathrm{vs} 74.50 \pm 29.52 \mathrm{ml} / \mathrm{min} / 1.73 \mathrm{~m}^{2}$ ) was lower than those in normo-uricemia patients (all $\mathrm{p}<0.05)$. When comparing immunological indexes of patients between the two groups, no significant difference was found in the levels of $\operatorname{IgA}, \operatorname{IgG}, \operatorname{IgM}$, and C3 (all p > 0.05). However, the level of C4 was significantly decreased in the normo-uricemia group $(p=0.003)$.

\section{The comparisons of Oxford classification for IgAN between two groups}

Histological features were evaluated using the MEST-C score of the 2016 updated Oxford Classification as Hernan et al. have reported (Trimarchi et al., 2017). The comparisons in histological manifestations between the hyperuricemia group and normo-uricemia group were shown in Figure 1. The M, E, and S scores in the two groups were similar( $\mathrm{p}>0.05)$. However, hyperuricemia patients had higher T scores than normo-uricemia patients $(1.00 \pm 0.87$ vs $0.64 \pm$ $0.76, \mathrm{p}=0.0023)$. In the previous Oxford study, crescent formation was not listed as an independent predictor of renal outcomes. However, Hernan Trimarchi and his working group 
157

158

159

160

161

162

163

164

165

166

167

168

169

170

171

172

173

174

175

176

177

178

179

180

181

supported crescents as a predictor of renal outcome and suggested that crescent (C) should be added to the MEST score. Therefore, we compared the crescent scores in two groups; no significant differences were found $(\mathrm{p}=0.4650)$. When comparing arterial lesions of patients in the two groups, hyperuricemia patients had higher scores of arteriosclerosis $(0.99 \pm 0.79$ vs 0.48 $\pm 0.71, \mathrm{p}<0.0001)$ and hyalinosis $(1.21 \pm 0.87 \mathrm{vs} 0.63 \pm 0.76, \mathrm{p}<0.0001)$ than those without. The glomerular sclerosis percentage in hyperuricemia patients was higher than that in normouricemia patients $(0.22 \pm 0.20$ vs $0.12 \pm 0.15, \mathrm{p}=0.0007)$.

\section{The comparison of different types of crescent lesions between the normo-uricemia group} and the hyperuricemia group

In the current investigation, results did not show any significant difference between the normouricemia group and hyperuricemia group in crescent (C) scores. Crescents could be divided into large and small crescents according to size and can be divided into cellular, fibrocellular and fibrous crescents according to crescent component (Jennette, 2003). Then we tried to explore whether SUA affected the formation of different types of crescents. As shown in Figure 2, patients with hyperuricemia had slightly higher percentages of total crescents, large crescents, and small crescents than normo-uricemia, however, there was no statistical difference $(0.10 \pm$ 0.14 vs $0.08 \pm 0.10,0.06 \pm 0.09$ vs $0.05 \pm 0.08,0.04 \pm 0.09$ vs $0.03 \pm 0.05$, respectively; all $p>$ 0.05). Our data also showed that participants with hyperuricemia had a slightly higher percentage of fibrocellular $(0.05 \pm 0.09$ vs $0.04 \pm 0.07, \mathrm{p}=0.1916)$ and fibrous crescent $(0.04 \pm 0.08$ vs 0.02 $\pm 0.05, \mathrm{p}=0.0837)$, while a significantly lower percentage of cellular $(0.005 \pm 0.02 \mathrm{vs} 0.020 \pm$ $0.05, \mathrm{p}=0.0024)$ compared to participants without hyperuricemia.

\section{The comparison of deposition of immune complexes and complements in renal biopsy} between the two groups

The pathogenesis of IgAN was considered to be associated with immunological and or genetic mechanisms. Therefore, we evaluated the deposition of immune complexes and complements in 
182 the two groups. We classified the immune complex deposition according to the fluorescence

183

184

185

186

187 intensity (FI) in this study. As shown in Figure 3, the deposition of IgG and FRA in hyperuricemia group was less than those in normo-uricemia group $(0.16 \pm 0.44$ vs $0.33 \pm 0.63$, $p$ $=0.0239 ; 0.27 \pm 0.51$ vs $0.45 \pm 0.55, \mathrm{p}=0.0258$, respectively). No significant difference was detected in IgA, IgM, and C3 deposition between the two groups (all p > 0.05).

\section{The value of the SUA level in predicting renal prognosis}

Finally, after an average of 25 months of follow-up, follow-up data were obtained from 193 patients. The endpoint of follow-up was defined as when patients needed dialysis treatment or when creatinine levels doubled. In this study, bout $70 \%$ of patients with HUA received uric acidlowering drugs during the follow-up. The renal survival curves according to the uric acid level suggested that patients with hyperuricemia have poorer renal outcome than patients without $(\mathrm{p}=$ 0.0147 , Figure 4A). And then, we plotted a ROC curve to estimate the value of SUA in predicting the prognosis of IgAN. For females, the optimal cutoff value was $361.1 \mu \mathrm{mol} / \mathrm{L}$, with sensitivity, specificity, and area under curve (AUC) of 0.7143, 0.7589, and 0.7679 \pm 0.08167 , respectively (Figure 4B). However, for males, the optimal cutoff value was $614 \mu \mathrm{mol} / \mathrm{L}$, with the sensitivity and specificity of 0.3333 and 0.9718 . The AUC for male patients was $0.5728 \pm 0.2029$ (Figure 4C). Our results revealed that the SUA level was a valuable indicator in predicting the prognosis of IgAN patients, especially in female patients, which is consistent with the research of Oh et al. (Oh et al. 2020).

\section{Discussion}

IgAN has a high incidence and a variety of clinical manifestations, from asymptomatic hematuria to progress to renal failure rapidly with a few months. Consequently, it is imperative to ascertain its influencing indicator, delay its progression, and prevent ESRD. A few studies have demonstrated that the SUA level is closely related to the progression of IgAN (Shi et al., 2012; Bakan et al., 2015; Cheng et al., 2013). However, there are still inconsistencies and even 
207

208

209

210

211

212

213

214

215

216

217

218

219

220

221

222

223

224

225

226

227

228

229

230

231

232

233

contradictions in some studies. Therefore, predicting the prognosis of IgAN remains difficult due to its diverse clinical features. In this retrospective study of 208 IgAN patients, we comprehensively evaluated the correlation between SUA levels and clinical and histopathological features. Our results revealed that the SUA level can be considered as a predictor for the prognosis of IgAN.

Results showed that patients with hyperuricemia have a poorer renal function and higher blood pressure, which is consistent with Cui et al. researched on 148 patients with IgAN (Cui et al., 2011). The results also revealed significant increases in blood pressure, lipid, C3, and C4 levels in patients with hyperuricemia. Previous studies have found that hyperuricemia is closely related to metabolic syndrome. Elevated SUA level has been confirmed to be an independent predictor of the development of diabetes, hypertension, hypertriglyceridemia, and nonalcoholic fatty liver disease (Lv et al., 2013; Grayson et al., 2011; Kuwabara et al., 2018; Yamada et al., 2010). Some studies also showed a significant improvement in homeostasis assessment for the IR index after lowering the level of SUA (Liu et al. 2015; Takir et al. 2015). Previous researches revealed that the levels of serum $\mathrm{C} 3$ and $\mathrm{C} 4$ positively correlated with the development of the metabolic syndrome and had been identified as important markers relevant to this disease (Meng et al., 2017; Meng et al., 2018; Xin et al., 2018). The increased activation products of C3 can accelerate the uptake of free fatty acids, the synthesis of triacylglycerol, and inhibit hormone-sensitive lipase in several adipocytes, thus contribute to the development of the metabolic syndrome (Phieler et al., 2013; Barbu et al., 2015). However, the mechanism of C4 in metabolic syndrome remains unclear.

To evaluate pathological changes, we classified patients using the 2016 update Oxford classification of IgAN as described above. We found that among patients with hyperuricemia, $32.86 \%$ had M, $28.57 \%$ had $\mathrm{S}$, and $35.71 \%$ had $\mathrm{E}$, which was higher than those in normouricemia patients, data were $29.71 \%, 27.54 \%, 30.43 \%$ respectively. However, no significant difference was discovered in $\mathrm{M}, \mathrm{E}$, and $\mathrm{S}$ scores between the hyperuricemia group and normouricemia group. As for the tubular atrophy/interstitial fibrosis, this study revealed that the $\mathrm{T}$ score 
234

235

236

237

238

239

240

241

242

243

244

245

246

247

248

249

250

251

252

253

254

255

256

257

258

259

260

of hyperuricemia patients was statistically higher than that of normo-uricemia patients. This result demonstrated that the level of SUA was related to tubular atrophy/interstitial fibrosis, and hyperuricemia can be used as a clinical marker for $\mathrm{T}$, which was consistent with the founding of Myllymaki et al. (Myllymaki et al., 2005) and Fan et al. (Fan et al. 2019). Recently, a study conducted by Nigro et al. found that the fractal dimension of tubules and the density of tubules negatively correlated the level of SUA and urea. They also suggested that SUA might be a better predictor to identify nephron integrity (Nigro et al. 2018). There were different views on the prognostic value of M, E, and S in different studies (Coppo et al., 2014b; Herzenberg et al., 2011b; Shi et al., 2011; Kang et al., 2012). Nevertheless, T was recognized as a prognostic indicator of IgAN in these studies. Thus, we regard the level of SUA as a valuable factor in predicting IgAN prognosis. The tubular atrophy/interstitial fibrosis caused by SUA might be explained by the following mechanisms. Urate crystals deposit in the renal tubules can directly damage or block the renal tubules, and also form uric acid renal stones to damage the kidney, which results in renal tubular atrophy and interstitial fibrosis, even renal failure (Viggiano et al. 2018). HUA could also promote the production of inflammatory factors such as MCP-1 and

TNF- $\beta 1$, which stimulates the inflammatory response and induce renal tubular injury and renal interstitial fibrosis (Romi et al. 2017). Moreover, HUA could decrease E-cadherin expression, increase $\alpha$-SMA expression, and induced tubular cells epithelial-mesenchymal transition, which results in renal tubulointerstitial injury (Liu et al. 2017).

Recently, the updated Oxford classification of IgAN recommends crescents (C) as a pathological predictor of renal outcomes in IgA nephropathy. In this current investigation, no correlation between the SUA level and C score was detected. Then, we compared the percentages of different types of $\mathrm{C}$ lesions in patients with hyperuricemia and those with normouricemia and found that the percentages of total crescents, large crescents, small crescents, fibrocellular crescents, and fibrous crescents were slightly higher in hyperuricemia patients, while the percentages of cell crescents were significantly lower. It is well known that the formation of the crescent is caused by the deposition of immune complexes, the activation of 
261

262

263

264

265

266

267

268

269

270

271

272

273

274

275

276

277

278

279

280

281

282

283

284

285

286

287

monocytes, the release of inflammatory cytokines, and the aggregation of fibrocytes. The previous report discovered that uric acid might promote the release of inflammatory cytokines such as TNF- $\alpha$, IL-1 $\beta$, IL-6 in IgA patients(Nakagawa et al., 2006). Therefore, we speculate that hyperuricemia may be involved in crescent formation by promoting the release of inflammatory cytokines.

As shown in Figure 2, hyperuricemia patients had significantly high percentages of glomerular sclerosis, including global glomerular sclerosis and ischemic glomerular sclerosis. Consequently, the level of SUA might indicate the severity of glomerular sclerosis. As reported in previous studies, many factors were related to glomerular sclerosis, including abnormal cytokine expression, podocyte injury or loss, and the activation of the RAS (Riser et al., 2000; Brown et al., 2000a; Brown et al., 2000b). As mentioned before, SUA could promote the release of inflammatory cytokines, and persistent inflammation may result in glomerular sclerosis. Furthermore, hyperuricemia may activate the renin-angiotensin system, cause glomerular hypertension, and reduce perfusion, which ultimately led to the formation of glomerular sclerosis (Sanchez-Lozada et al., 2005). Besides, the uric acid can induce cellular oxidation through the xanthine oxidase pathway and then result in podocyte damage, which might be another cause of glomerular sclerosis.

We also evaluated the effect of elevated uric acid on vascular pathological changes, including arteriosclerosis and arteriolar hyalinosis. We found that the vascular lesions were more severe in hyperuricemia patients, and several mechanisms could explain this difference. Firstly, the elevated uric acid levels could inhibit nitric oxide synthase, induce inflammatory reactions, activate the renin-angiotensin system, cause endothelial cells dysfunction, and eventually lead to the lesions of vascular (Behradmanesh et al., 2013; Corry et al., 2008). Secondly, increased serum lipid levels and oxidation of LDL can promote the progression of atherosclerosis and arteriolar hyalinosis.

An early study by Kim SJ et al, which included 343 patients with IgAN found that the decreased serum $\mathrm{C} 3$ level and the deposition of $\mathrm{C} 3$ in mesangial could independently predict the 
288

289

290

291

292

293

294

295

296

297

298

299

300

301

302

303

304

305

306

307

308

309

310

311

progression of $\operatorname{IgAN}$, indicating that the activation of complements was closely related to the IgAN pathogenesis (Kim et al., 2012). However, our result showed there was no significant difference in the deposition of IgA, IgM, and C3 between patients with hyperuricemia and those without. This could be due to the small sample size or different races of the study.

There are several limitations in this study. On the one hand, the sample size of this pilot study was relatively small. Next, we will conduct a multicenter, large sample size prospective study for further analysis. On the other hand, further studies are required to explore whether lowering uric acid levels could improve the outcome of IgAN.

In conclusion, the SUA level affects renal function and pathophysiology of IgAN. The levels of SUA can be considered as a prognostic indicator for IgAN, which is of great significance in guiding treatment decisions and assessing prognosis.

\section{Funding}

This study was supported by the National Natural Science Foundation of China (No.81770686, $81970591)$ and the independent innovation research funds project of Huazhong University of Science and Technology (No.2017KFYXJJ101).

\section{Competing Interests}

The authors declare there are no competing interests.

\section{References}

Bakan A, Oral A, Elcioglu OC, Takir M, Kostek O, Ozkok A, Basci S, Sumnu A, Ozturk S, Sipahioglu M, Turkmen A, Voroneanu L, Covic A, and Kanbay M. 2015. Hyperuricemia is associated with progression of IgA nephropathy. International urology and nephrology 47:673678. 10.1007/s11255-015-0939-7

Barbour, S., and Reich, H. 2018. An update on predicting renal progression in IgA nephropathy. Current opinion in nephrology and hypertension:1. DOI:10.1097/MNH.0000000000000405 
312 Barbour, S.J., Espino-Hernandez, G., Reich, H.N., Coppo, R., Roberts, I.S., Feehally, J., 313 Herzenberg, A.M., and Cattran, D.C. 2016. The MEST score provides earlier risk prediction in 314 IgA nephropathy. Kidney international 89:167-175. DOI:10.1038/ki.2015.322

315 Barbour, S.J., and Reich, H.N. 2012. Risk stratification of patients with IgA nephropathy. 316 American journal of kidney diseases 59:865-873. DOI: 10.1053/j.ajkd.2012.02.326

317 Barbu, A., Hamad, O.A., Lind, L., Ekdahl, K.N., and Nilsson, B. 2015. The role of complement 318 factor C3 in lipid metabolism. Molecular immunology 67:101-107.

319 DOI: 10.1016/j.molimm.2015.02.027

320 Behradmanesh, S., Horestani, M.K., Baradaran, A., and Nasri, H. 2013. Association of serum 321 uric acid with proteinuria in type 2 diabetic patients. Journal of Research in Medical Sciences $322 \quad 18: 44-46$.

323 Braga F, Pasqualetti S, Ferraro S, and Panteghini M. 2016. Hyperuricemia as risk factor for 324 coronary heart disease incidence and mortality in the general population: a systematic review and meta-analysis. Clinical chemistry and laboratory medicine 54. DOI: 10.1515/cclm-2015-0523.

326 Brown, N.J., Nakamura, S., Ma, L., Nakamura, I., Donnert, E., Freeman, M., Vaughan, D.E., and 327 Fogo, A.B. 2000a. Aldosterone modulates plasminogen activator inhibitor-1 and 328 glomerulosclerosis in vivo. Kidney international 58:1219-1227. DOI:10.1046/j.1523-1755.2000. 00277.x

330 Cheng, G., Liu, D., Zhang, N., Tang, L., Zhao, Z., and Liu, Z. 2013. Clinical and prognostic 331 implications of serum uric acid levels on IgA nephropathy: a cohort study of 348 cases with a 332 mean 5-year follow-up. Clinical nephrology 80:40-46. DOI:10.5414/CN107813

333 Clarson LE, Chandratre P, Hider SL, Belcher J, Heneghan C, Roddy E, and Mallen CD. 2015. 334 Increased cardiovascular mortality associated with gout: a systematic review and meta-analysis. 335 European journal of preventive cardiology 22:335-343. DOI: 10.1177/2047487313514895.

336 Coppo, R., Troyanov, S., Bellur, S., Cattran, D., Cook, H.T., Feehally, J., Roberts, I.S., Morando, 337 L., Camilla, R., Tesar, V., Lunberg, S., Gesualdo, L., Emma, F., Rollino, C., Amore, A., Praga, 338 M., Feriozzi, S., Segoloni, G., Pani, A., Cancarini, G., Durlik, M., Moggia, E., Mazzucco, G., 
339 Giannakakis, C., Honsova, E., Sundelin, B.B., Di Palma, A.M., Ferrario, F., Gutierrez, E., 340 Asunis, A.M., Barratt, J., Tardanico, R., and Perkowska-Ptasinska, A. 2014a. Validation of the

341 Oxford classification of IgA nephropathy in cohorts with different presentations and treatments.

342 Kidney international 86:828-836. DOI:10.1038/ki.2014.63

343 Coppo, R., and D'Amico, G. 2005. Factors predicting progression of IgA nephropathies. Journal 344 of nephrology 18:503-512.

345 Corry, D.B., Eslami, P., Yamamoto, K., Nyby, M.D., Makino, H., and Tuck, M.L. 2008. Uric 346 acid stimulates vascular smooth muscle cell proliferation and oxidative stress via the vascular 347 renin-angiotensin system. Journal of hypertension 26:269-275.

348 DOI:10.1097/HJH.0b013e3282f240bf

349 Cui, M.J., Zhang, B.H., Xiao, Q.F., Zhu, F.L., and Wang, H.Y. 2011. The relationship between 350 hyperuricemia and clinic pathology of IgA nephropathy. Zhonghua Nei Ke Za Zhi 50:659-663. 351 D'Amico, G. 2004. Natural history of idiopathic IgA nephropathy and factors predictive of 352 disease outcome. Seminars in nephrology 24:179-196.

353 Fan S, Zhang P, Wang AY, Wang X, Wang L, Li G, and Hong D. 2019. Hyperuricemia and its 354 related histopathological features on renal biopsy. BMC nephrology 20:95.

355 DOI: $10.1186 / \mathrm{s} 12882-019-1275-4$

356 Feig DI. 2014. Serum uric acid and the risk of hypertension and chronic kidney disease. Current 357 opinion in rheumatology 26:176-185. DOI: 10.1097/BOR.0000000000000033

358 Ghane, S.F., and Assadi, F. 2018. Effect of allopurinol on the glomerular filtration rate of 359 children with chronic kidney disease. PEDIATRIC NEPHROLOGY. DOI:10.1007/s00467-018$360 \quad 3943-1$

361 Grayson, P.C., Kim, S.Y., LaValley, M., and Choi, H.K. 2011. Hyperuricemia and incident 362 hypertension: a systematic review and meta-analysis. Arthritis Care Res (Hoboken) 63:102-110. 363 DOI:10.1002/acr.20344

364 Gustafsson D, and Unwin R. 2013. The pathophysiology of hyperuricaemia and its possible 365 relationship to cardiovascular disease, morbidity and mortality. BMC nephrology 14:164. 
366

367

368

369

370

371

372

373

374

375

376

377

378

379

380

381

382

383

384

385

386

387

388

389

390

391

392
$10.1186 / 1471-2369-14-164$

Herzenberg, A.M., Fogo, A.B., Reich, H.N., Troyanov, S., Bavbek, N., Massat, A.E., Hunley, T.E., Hladunewich, M.A., Julian, B.A., Fervenza, F.C., and Cattran, D.C. 2011a. Validation of the Oxford classification of IgA nephropathy. Kidney international 80:310-317.

DOI:10.1038/ki.2011.126

Jennette, J.C. 2003. Rapidly progressive crescentic glomerulonephritis. Kidney international 63:1164-1177. DOI:10.1046/j.1523-1755.2003. 00843.x

Kang, S.H., Choi, S.R., Park, H.S., Lee, J.Y., Sun, I.O., Hwang, H.S., Chung, B.H., Park, C.W., Yang, C.W., Kim, Y.S., Choi, Y.J., and Choi, B.S. 2012. The Oxford classification as a predictor of prognosis in patients with IgA nephropathy. Nephrol Dial Transplant 27:252-258.

DOI:10.1093/ndt/gfr295

Katafuchi, R., Ninomiya, T., Nagata, M., Mitsuiki, K., and Hirakata, H. 2011. Validation study of oxford classification of IgA nephropathy: the significance of extracapillary proliferation. Clin J Am Soc Nephrol 6:2806-2813. DOI:10.2215/CJN.02890311

Kim, S.J., Koo, H.M., Lim, B.J., Oh, H.J., Yoo, D.E., Shin, D.H., Lee, M.J., Doh, F.M., Park, J.T., Yoo, T.H., Kang, S.W., Choi, K.H., Jeong, H.J., and Han, S.H. 2012. Decreased circulating C3 levels and mesangial C3 deposition predict renal outcome in patients with IgA nephropathy. PLoS One 7: e40495. DOI: 10.1371/journal.pone.0040495

Kim SY, Guevara JP, Kim KM, Choi HK, Heitjan DF, and Albert DA. 2010. Hyperuricemia and coronary heart disease: a systematic review and meta-analysis. Arthritis care \& research 62:170180. DOI: $10.1002 /$ acr.20065

Kuwabara, M., Borghi, C., Cicero, A., Hisatome, I., Niwa, K., Ohno, M., Johnson, R.J., and Lanaspa, M.A. 2018. Elevated serum uric acid increases risks for developing high LDL cholesterol and hypertriglyceridemia: A five-year cohort study in Japan. International journal of cardiology 261:183-188. DOI: 10.1016/j.ijcard.2018.03.045

Le, W., Liang, S., Hu, Y., Deng, K., Bao, H., Zeng, C., and Liu, Z. 2012. Long-term renal survival and related risk factors in patients with IgA nephropathy: results from a cohort of 1155

Peer] reviewing PDF | (2020:05:48827:2:0:NEW 12 Sep 2020) 
393

394

395

396

397

398

399

400

401

402

403

404

405

406

407

408

409

410

411

412

413

414

415

416

417

418

419

cases in a Chinese adult population. Nephrology dialysis transplantation 27:1479-1485.

DOI:10.1093/ndt/gfr527

Li M, Hou W, Zhang X, Hu L, and Tang Z. 2014. Hyperuricemia and risk of stroke: a systematic review and meta-analysis of prospective studies. Atherosclerosis 232:265-270.

DOI: $10.1016 /$ j.atherosclerosis.2013.11.051

Liu H, Xiong J, He T, Xiao T, Li Y, Yu Y, Huang Y, Xu X, Huang Y, Zhang J, Zhang B, and Zhao J. 2017. High Uric Acid-Induced Epithelial-Mesenchymal Transition of Renal Tubular Epithelial Cells via the TLR4/NF-kB Signaling Pathway. American journal of nephrology 46:333-342. DOI:10.1159/000481668

Liu P, Wang H, Zhang F, Chen Y, Wang D, and Wang Y. 2015. The Effects of Allopurinol on the Carotid Intima-media Thickness in Patients with Type 2 Diabetes and Asymptomatic Hyperuricemia: A Three-year Randomized Parallel-controlled Study. Intern Med 54:2129-2137. DOI: $10.2169 /$ internalmedicine.54.4310

Lv, J., Shi, S., Xu, D., Zhang, H., Troyanov, S., Cattran, D.C., and Wang, H. 2013. Evaluation of the Oxford Classification of IgA nephropathy: a systematic review and meta-analysis. American journal of kidney diseases 62:891-899. DOI: 10.1053/j.ajkd.2013.04.021

Lv, Q., Meng, X.F., He, F.F., Chen, S., Su, H., Xiong, J., Gao, P., Tian, X.J., Liu, J.S., Zhu, Z.H., Huang, K., and Zhang, C. 2013. High serum uric acid and increased risk of type 2 diabetes: a systemic review and meta-analysis of prospective cohort studies. PLoS One 8: e56864. DOI: 10.1371/journal.pone.0056864

Maixnerova, D., Reily, C., Bian, Q., Neprasova, M., Novak, J., and Tesar, V. 2016. Markers for the progression of IgA nephropathy. Journal of nephrology 29:535-541. DOI:10.1007/s40620016-0299-0

Mariani, G., Freitas, L., Zollner, R.L., and Ribeiro, A.M. 2018. Renal outcome in IgA nephropathy according to Oxford classification and ultrastructural analysis in a Brazilian center. Clinical nephrology 89:270-276. DOI:10.5414/CN109062

Meng, G., Li, H., Li, Y., Zhang, Q., Liu, L., Wu, H., Xia, Y., Bao, X., Gu, Y., Su, Q., Fang, L., 
420 Yang, H., Yu, F., Shi, H., Sun, S., Wang, X., Zhou, M., Jia, Q., Song, K., Chang, H., Wu, Y., and 421 Niu, K. 2018. Sex-Specific Associations Between Complement Component 3 and Component 4 422 Levels and Metabolic Syndrome in an Adult Population. Metab Syndr Relat Disord 16:143-149. 423 DOI:10.1089/met.2017.0111

424 Meng, G., Zhu, Q., Shao, J., Zhang, Q., Liu, L., Wu, H., Xia, Y., Bao, X., Gu, Y., Wang, H., Shi, 425 H., Sun, S., Wang, X., Zhou, M., Jia, Q., Wang, G., Song, K., Wu, Y., and Niu, K. 2017.

426 Comparing the diagnostic ability of inflammatory markers in metabolic syndrome. Clinica 427 chimica acta 475:1-6. DOI: 10.1016/j.cca.2017.09.023

428 Moriyama T, Itabashi M, Takei T, Kataoka H, Sato M, Shimizu A, Iwabuchi Y, Nishida M, 429 Uchida K, and Nitta K. 2015. High uric acid level is a risk factor for progression of IgA 430 nephropathy with chronic kidney disease stage G3a. Journal of nephrology 28:451-456.

431 DOI: $10.1007 / \mathrm{s} 40620-014-0154-0$

432 Myllymaki, J., Honkanen, T., Syrjanen, J., Helin, H., Rantala, I., Pasternack, A., and Mustonen, J. 433 2005. Uric acid correlates with the severity of histopathological parameters in IgA nephropathy.

434 Nephrology dialysis transplantation 20:89-95. DOI:10.1093/ndt/gfh584

435 Nagasawa Y, Yamamoto R, Shoji T, Shinzawa M, Hasuike Y, Nagatoya K, Yamauchi A, 436 Hayashi T, Kuragano T, Moriyama T, Isaka Y, and Nakanishi T. 2016. Serum Uric Acid Level 437 Predicts Progression of IgA Nephropathy in Females but Not in Males. PLoS One 11:e0160828. 438 DOI: 10.1371/journal.pone.0160828

439 Nakagawa, T., Kang, D.H., Feig, D., Sanchez-Lozada, L.G., Srinivas, T.R., Sautin, Y., Ejaz, 440 A.A., Segal, M., and Johnson, R.J. 2006. Unearthing uric acid: an ancient factor with recently 441 found significance in renal and cardiovascular disease. Kidney international 69:1722-1725.

442 DOI:10.1038/sj.ki.5000391

443 Nigro M, Viggiano D, Ragone V, Trabace T, di Palma A, Rossini M, Capasso G, Gesualdo L, 444 and Gigliotti G. 2018. A cross-sectional study on the relationship between hematological data 445 and quantitative morphological indices from kidney biopsies in different glomerular diseases. 446 BMC nephrology 19:62. DOI: 10.1186/s12882-018-0846-0 
447 Oh TR, Choi HS, Kim CS, Kang KP, Kwon YJ, Kim SG, Ma SK, Kim SW, and Bae EH. 2020.

448 The Effects of Hyperuricemia on the Prognosis of IgA Nephropathy are More Potent in Females.

449 Journal of clinical medicine 9. DOI: 10.3390/jcm9010176

450 Phieler, J., Garcia-Martin, R., Lambris, J.D., and Chavakis, T. 2013. The role of the complement

451 system in metabolic organs and metabolic diseases. SEMINARS IN IMMUNOLOGY 25:47-53.

452 DOI: 10.1016/j.smim.2013.04.003

453 Riser, B.L., Cortes, P., and Yee, J. 2000. Modelling the effects of vascular stress in mesangial

454 cells. Curr Opin Nephrol Hypertens 9:43-47

455 Romi MM, Arfian N, Tranggono U, Setyaningsih WAW, and Sari DCR. 2017. Uric acid causes

456 kidney injury through inducing fibroblast expansion, Endothelin-1 expression, and inflammation.

457 BMC nephrology 18:326. DOI: 10.1186/s12882-017-0736-X

458 Sanchez-Lozada, L.G., Tapia, E., Santamaria, J., Avila-Casado, C., Soto, V., Nepomuceno, T.,

459 Rodriguez-Iturbe, B., Johnson, R.J., and Herrera-Acosta, J. 2005. Mild hyperuricemia induces

460 vasoconstriction and maintains glomerular hypertension in normal and remnant kidney rats.

461 Kidney international 67:237-247. DOI:10.1111/j.1523-1755.2005. 00074.x

462 Shi, S.F., Wang, S.X., Jiang, L., Lv, J.C., Liu, L.J., Chen, Y.Q., Zhu, S.N., Liu, G., Zou, W.Z.,

463 Zhang, H., and Wang, H.Y. 2011. Pathologic predictors of renal outcome and therapeutic

464 efficacy in IgA nephropathy: validation of the oxford classification. Clin J Am Soc Nephrol

465 6:2175-2184. DOI:10.2215/CJN.11521210

466 Shi, Y., Chen, W., Jalal, D., Li, Z., Chen, W., Mao, H., Yang, Q., Johnson, R.J., and Yu, X. 2012.

467 Clinical Outcome of Hyperuricemia in IgA Nephropathy: A Retrospective Cohort Study and

468 Randomized Controlled Trial. Kidney and Blood Pressure Research 35:153-160

469 DOI:10.1159/000331453

470 Su HY, Yang C, Liang D, and Liu HF. 2020. Research Advances in the Mechanisms of

471 Hyperuricemia-Induced Renal Injury. Biomed Res Int 2020:5817348.

472 DOI: $10.1155 / 2020 / 5817348$

473 Sulikowska, B., Manitius, J., Odrowąż-Sypniewska, G., Łysiak-Szydłowska, W., and Rutkowski, 
474

475

476

477

478

479

480

481

482

483

484

485

486

487

488

489

490

491

492

493

494

495

496

497

498

499

500

B. 2008. Uric Acid Excretion and Dopamine-Induced Glomerular Filtration Response in Patients with IgA Glomerulonephritis. American journal of Nephrology 28:391-396.

DOI:10.1159/000112271

Takir M, Kostek O, Ozkok A, Elcioglu OC, Bakan A, Erek A, Mutlu HH, Telci O, Semerci A, Odabas AR, Afsar B, Smits G, Alanaspa M, Sharma S, Johnson RJ, and Kanbay M. 2015. Lowering Uric Acid With Allopurinol Improves Insulin Resistance and Systemic Inflammation in Asymptomatic Hyperuricemia. Journal of investigative medicine: the official publication of the American Federation for Clinical Research 63:924-929. DOI:

10.1097/JIM.0000000000000242

Trimarchi, H., Barratt, J., Cattran, D.C., Cook, H.T., Coppo, R., Haas, M., Liu, Z.H., Roberts, I.S., Yuzawa, Y., Zhang, H., and Feehally, J. 2017. Oxford Classification of IgA nephropathy 2016: an update from the IgA Nephropathy Classification Working Group. Kidney international 91:1014-1021. DOI: 10.1016/j.kint.2017.02.003

Viggiano D, Gigliotti G, Vallone G, Giammarino A, Nigro M, and Capasso G. 2018. UrateLowering Agents in Asymptomatic Hyperuricemia: Role of Urine Sediment Analysis and Musculoskeletal Ultrasound. Kidney \& blood pressure research 43:606-615.

DOI: $10.1159 / 000489145$

Wang J, Qin T, Chen J, Li Y, Wang L, Huang H, and Li J. 2014. Hyperuricemia and risk of incident hypertension: a systematic review and meta-analysis of observational studies. PLoS One 9:e114259. DOI: 10.1371/journal.pone.0114259

Xin, Y., Hertle, E., van der Kallen, C., Schalkwijk, C.G., Stehouwer, C., and van Greevenbroek, M. 2018. Complement $\mathrm{C} 3$ and $\mathrm{C} 4$, but not their regulators or activated products, are associated with incident metabolic syndrome: the CODAM study. Endocrine 62:617-627.

DOI:10.1007/s12020-018-1712-3

Yamada, T., Suzuki, S., Fukatsu, M., Wada, T., Yoshida, T., and Joh, T. 2010. Elevated serum uric acid is an independent risk factor for nonalcoholic fatty liver disease in Japanese undergoing a health checkup. Acta Gastroenterol Belg 73:12-17. 
501 Zhu B, Yu D-R, Lv J-C, Lin Y, Li Q, Yin J-Z, Du Y-Y, Tang X-L, Mao L-C, Li Q-F, Sun Y, Liu 502 L, Li X-F, Fei D, Wei X-Y, Zhu C-F, Cheng X-X, Chen H-Y, and Wang Y-J. 2018. Uric Acid as 503 a Predictor of Immunoglobulin A Nephropathy Progression: A Cohort Study of 1965 Cases. 504 American journal of nephrology 48:127-136. DOI: 10.1159/000489962

505 Zhou, J., Chen, Y., Liu, Y., Shi, S., Li, X., Wang, S., and Zhang, H. 2014. Plasma uric acid level 506 indicates tubular interstitial leisions at early stage of IgA nephropathy. BMC Nephrology 15:11. 507 DOI:10.1186/1471-2369-15-11

508 


\section{Table 1 (on next page)}

The histopathological grading schema in IgAN. 


\begin{tabular}{|c|c|}
\hline Histopathological features & Definition \\
\hline \multicolumn{2}{|l|}{ Mesangial hypercellularity } \\
\hline M0 & Mesangial score ${ }^{\mathrm{a}} \leq 0.5$ \\
\hline M1 & Mesangial score $>0.5$ \\
\hline \multicolumn{2}{|c|}{ Endocapillary hypercellularity } \\
\hline E0 & absent \\
\hline E1 & present \\
\hline \multicolumn{2}{|c|}{ Segmental glomerulosclerosis } \\
\hline S0 & absent \\
\hline S1 & present \\
\hline \multirow{2}{*}{\multicolumn{2}{|c|}{$\begin{array}{l}\text { Tubular atrophy/interstitial } \\
\text { fibrosis }\end{array}$}} \\
\hline & \\
\hline T0 & $\leq 25 \%$ \\
\hline T1 & $26 \%-50 \%$ \\
\hline $\mathrm{T} 2$ & $>50 \%$ \\
\hline \multicolumn{2}{|l|}{ Crescents } \\
\hline $\mathrm{C} 0$ & absent \\
\hline $\mathrm{C} 1$ & present in $\geq 1$ glomerulus \\
\hline $\mathrm{C} 2$ & in $>25 \%$ of glomeruli \\
\hline \multicolumn{2}{|l|}{ Arteriosclerosis } \\
\hline 0 & no intimal thickening \\
\hline 1 & intimal thickened and $<$ thickness of media \\
\hline 2 & intimal thickened and $>$ thickness of media \\
\hline \multicolumn{2}{|l|}{ Arteriolar hyalinosis } \\
\hline 0 & absent \\
\hline 1 & $1-25 \%$ of arterioles present \\
\hline 2 & $26 \%-50 \%$ of arterioles present \\
\hline 3 & $>50 \%$ of arterioles present \\
\hline
\end{tabular}

2 Notes. a. The glomerular mesangial area is the area between the glomerular

3 capillary loops and is composed of mesangial cells and mesangial matrix.

4 The mesangial hypercellularity score is calculated according to the mesangial cell

5 count in per mesangial area: the scores of $<4,4-5,6-7, \geq 8$ mesangial cells are 0 ,

$61,2,3$, respectively. The mean score of all glomerulus is the mesangial

7 hypercellularity score. 
Table 2 (on next page)

The demographic and clinical data of patients. 


\begin{tabular}{lc}
\hline Parameters & Results \\
\hline Age (years; mean \pm SD) & $33.8 \pm 10.7$ \\
\hline Gender (male/female) & $82 / 126$ \\
\hline mean arterial pressure (mm Hg; mean \pm SD) & $94.0 \pm 11.7$ \\
\hline Treated with antihypertensive drugs, n (\%) & $22(10.58 \%)$ \\
\hline Taking ACEI and/or ARB, n (\%) & $30(14.42 \%)$ \\
\hline Underwent tonsillectomy, n (\%) & $10(4.81 \%)$ \\
\hline Hematuria, n (\%) & $207(99.52 \%)$ \\
\hline Albumin (g/l; mean \pm SD) & $39.48 \pm 5.77$ \\
\hline Uric acid ( $\boldsymbol{\mu m o l / l ; ~ m e a n ~} \pm$ SD) & $348.00 \pm 109.10$ \\
\hline Serum creatinine $(\boldsymbol{\mu m o l} / \mathbf{l} ;$ mean \pm SD) & $89.89 \pm 41.49$ \\
\hline eGFR (ml/min per 1.73 m2; mean \pm SD) & $82.23 \pm 30.22$ \\
\hline 24-h urine protein (g/d; mean \pm SD) & $1.35 \pm 1.58$ \\
\hline
\end{tabular}

2 Notes. eGFR was calculated with the CKD-EPI equation. Data are expressed as mean \pm SD 3 or number (\%). 
Table 3(on next page)

The comparison of clinical characteristics between normo-uricemia group and hyperuricemia group. 


\begin{tabular}{|c|c|c|c|}
\hline Parameters & Normo-uricemia & Hyperuricemia & $\mathbf{P}$ \\
\hline Patients(n) & 138 & 70 & - \\
\hline Gender (male/female) & $46 / 92$ & $36 / 34$ & 0.012 \\
\hline Age (years) & $33.7 \pm 10.5$ & $34.2 \pm 11.2$ & 0.7311 \\
\hline Body weight (kg) & $59.7 \pm 9.3$ & $64.8 \pm 12.9$ & 0.0057 \\
\hline MAP (mm Hg) & $91.8 \pm 10.7$ & $98.2 \pm 12.5$ & 0.0002 \\
\hline White blood cells (*10^9/l) & $7.04 \pm 1.85$ & $7.55 \pm 2.44$ & 0.1248 \\
\hline Hemoglobin (g/l) & $129.60 \pm 16.75$ & $130.30 \pm 23.52$ & 0.8214 \\
\hline Platelet $\left({ }^{\left.* 10^{\wedge} 9 / \mathrm{l}\right)}\right.$ & $237.50 \pm 62.07$ & $239.10 \pm 70.38$ & 0.8704 \\
\hline APTT(s) & $37.43 \pm 3.66$ & $37.57 \pm 3.23$ & 0.7833 \\
\hline FDP (mg/l) & $3.23 \pm 0.74$ & $3.86 \pm 1.38$ & 0.0006 \\
\hline Total protein(g/l) & $68.84 \pm 7.08$ & $67.80 \pm 8.40$ & 0.3497 \\
\hline $\operatorname{Albumin}(g / l)$ & $40.03 \pm 4.94$ & $38.40 \pm 7.05$ & 0.0868 \\
\hline Total cholesterol(mmol/l) & $4.53 \pm 1.17$ & $5.01 \pm 1.55$ & 0.0260 \\
\hline Triglyceride(mmol/l) & $1.63 \pm 1.02$ & $2.54 \pm 1.89$ & 0.0005 \\
\hline HDL-cholesterol (mmol/l) & $1.34 \pm 0.49$ & $1.14 \pm 0.35$ & 0.0056 \\
\hline LDL-cholesterol (mmol/l) & $2.73 \pm 0.95$ & $3.03 \pm 1.41$ & 0.1606 \\
\hline Uric acid $(\mu \mathrm{mol} / \mathrm{l})$ & $290.00 \pm 66.27$ & $462.40 \pm 83.46$ & $<0.0001$ \\
\hline Blood urea nitrogen $(\mathrm{mmol} / \mathrm{l})$ & $5.12 \pm 1.52$ & $6.49 \pm 1.95$ & $<0.0001$ \\
\hline Serum creatinine $(\mu \mathrm{mol} / \mathrm{l})$ & $80.40 \pm 40.58$ & $109.00 \pm 36.54$ & $<0.0001$ \\
\hline eGFR $(\mathrm{ml} / \mathrm{min}$ per $1.73 \mathrm{~mm} 2)$ & $98.98 \pm 25.12$ & $74.50 \pm 29.52$ & $<0.0001$ \\
\hline 24-hour urine protein $(\mathrm{g} / \mathrm{d})$ & $1.05 \pm 1.27$ & $1.81 \pm 1.67$ & 0.0015 \\
\hline $\operatorname{IgA}(g / \mathbf{l})$ & $3.22 \pm 1.15$ & $3.11 \pm 1.00$ & 0.5328 \\
\hline $\operatorname{IgG}(g / \mathrm{l})$ & $2.91 \pm 0.26$ & $2.95 \pm 0.38$ & 0.1506 \\
\hline $\operatorname{IgM}(g / l)$ & $1.56 \pm 1.19$ & $1.42 \pm 0.67$ & 0.3222 \\
\hline C3(g/l) & $0.90 \pm 0.22$ & $1.00 \pm 0.24$ & 0.0055 \\
\hline $\mathrm{C} 4(\mathrm{~g} / \mathrm{l})$ & $0.20 \pm 0.07$ & $0.25 \pm 0.12$ & 0.0030 \\
\hline
\end{tabular}

2

Notes. MAP, mean arterial pressure; APTT, activated partial thromboplastin time; FDP, fibrin degradation products. eGFR was calculated with the CKD-EPI equation. Bold Pvalues indicate a statistical significance of $P<0.05$. 


\section{Figure 1}

Figure 1. The comparisons of Oxford classification and arterial lesions between normouricemia and hyperuricemia patients in IgA nephropathy.

(A) mesangial hypercellularity scores; (B) endocapillary hypercellularity scores; (C) segmental sclerosis scores; (D) tubular atrophy / interstitial fibrosis scores; (E) crescent scores; (F) MEST-C total scores; (G) arteriosclerosis score; $(\mathrm{H})$ hyalinosis score; (I) the percent of glomerular sclerosis.
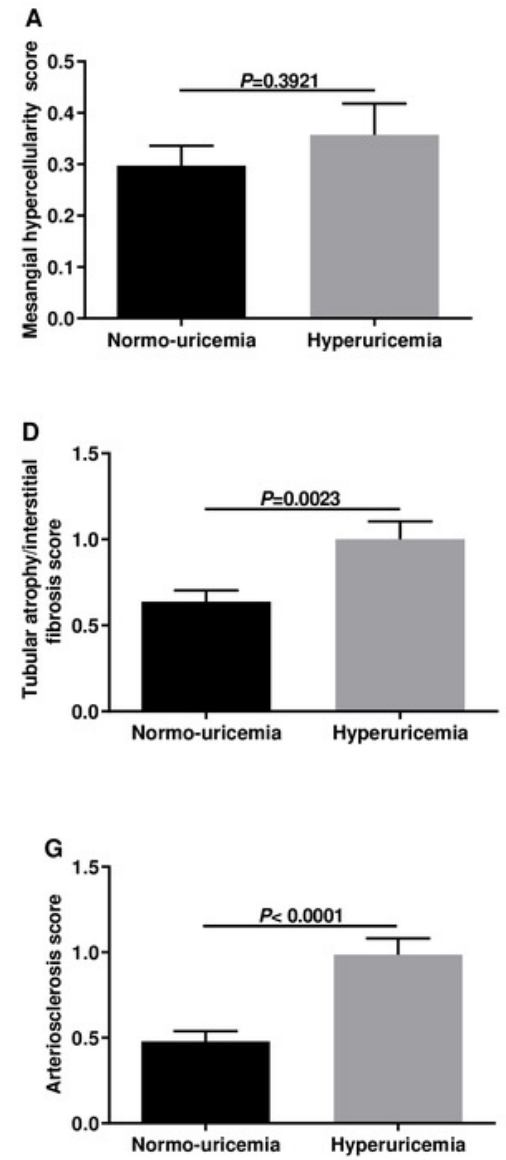
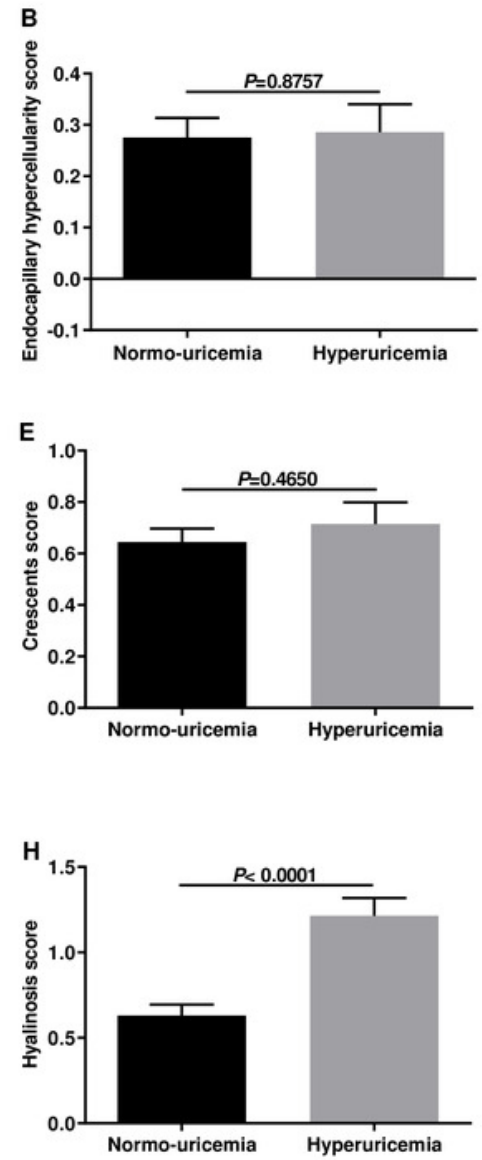
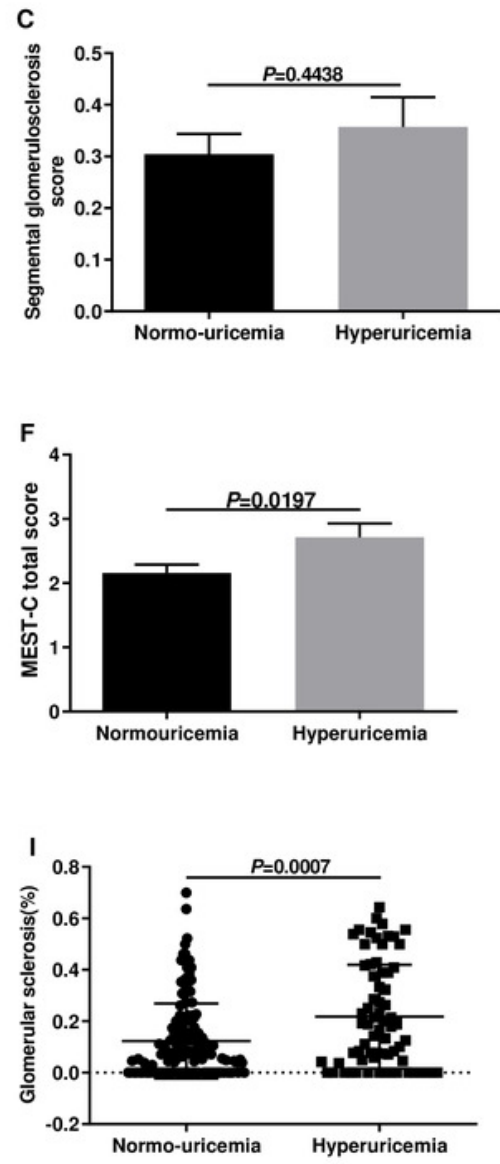
Figure 2

Figure 2. Percentages of different types of crescents among the IgAN patients with normo-uricemia or hyperuricemia.

The proportion of total crescents (A), large crescents (B), small crescents (C), cellular crescents (D), fibrocellular crescents (E), and fibrous crescents (F).
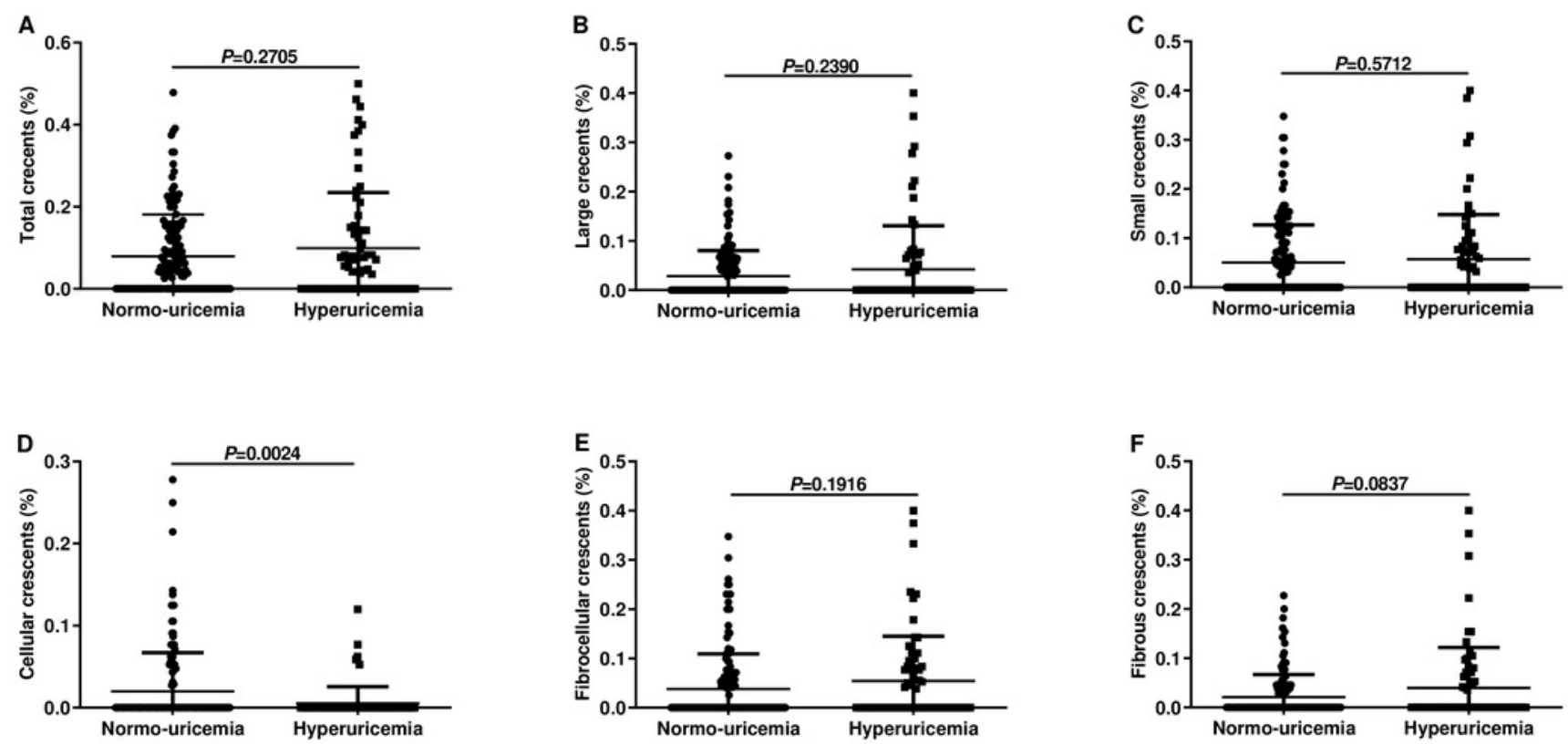
Figure 3

Figure 3. The fluorescence intensity scores of immune complex deposits in renal biopsy.

Fluorescence intensity scores of IgA deposition (A), IgG deposition (B), IgM deposition(C), C3 deposition (D), and FR A deposition(E).
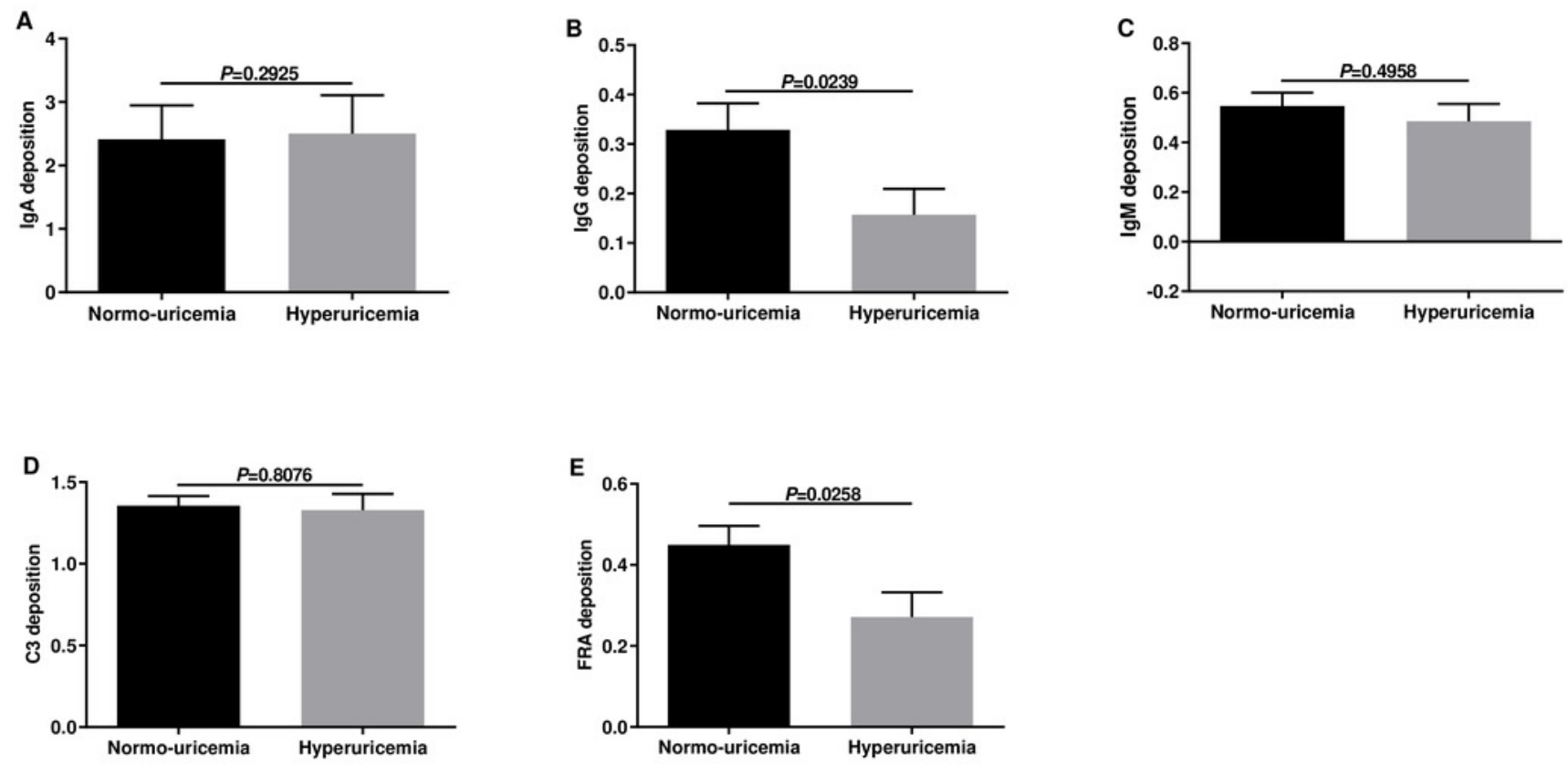
Figure 4

Figure 4. The value of the serum uric acid level in predicting renal prognosis.

The overall renal survival rate of $\operatorname{IgAN}(\mathrm{A})$. ROC curve analysis evaluates the predictive value of uric acid level for the poor renal outcomes in females (B)and in males(C) with IgAN.
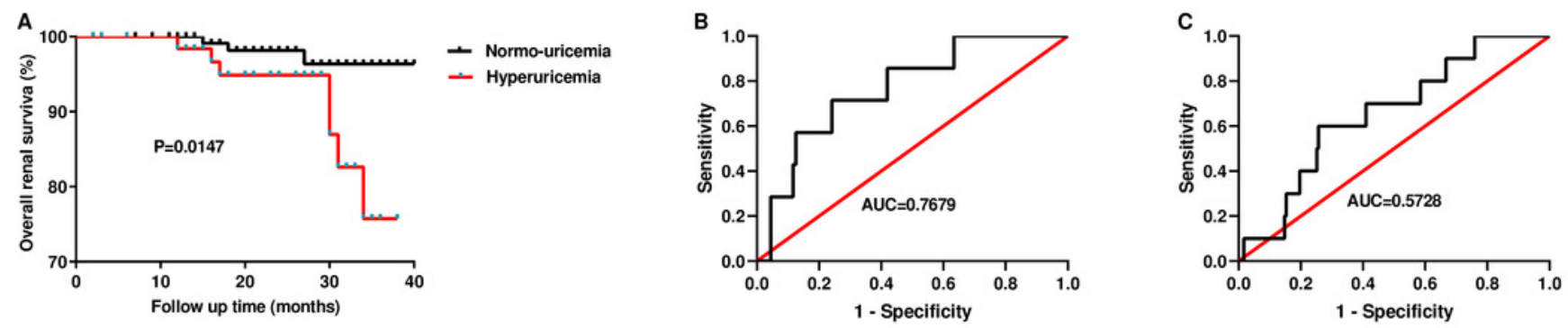